\title{
Pneumonia Detection and Classification Using Deep Learning on Chest $\mathrm{X}$-Ray Images
}

\author{
Muazzez Buket DARICI*1, Zumray DOKUR², Tamer OLMEZ ${ }^{3}$
}

\begin{abstract}
Submitted: 24/09/2020 Accepted : 23/11/2020
Abstract: Pneumonia is a bacterial infection caused people of all ages with mild to severe inflammation of the lung tissue. The best known and most common clinical method for the diagnosis of pneumonia is chest X-ray imaging. But the diagnosis of pneumonia from chest Xray images is a difficult task, even for specialist radiologists. In developing countries, this lung disease becomes one of the deadliest among children under the age of 5 and causing $15 \%$ of deaths recorded annually. Therefore, in this study, firstly the presence of the disease was tried to be determined using chest X-ray dataset. In addition, using the bacterial and viral pneumonia classes which are two different types of pneumonia, multi class classification which consists of viral pneumonia, bacterial pneumonia and healthy has been done. Since the used dataset does not have a balanced distribution among all classes, SMOTE (Synthetic Minority Over-sampling Technique) method has been used to deal with imbalanced dataset. CNN model and models in Ensemble Learning have been created from scratch instead of using weights of pre-trained networks to see the effectiveness of CNN weights on medical data. For each classification problem, two different deep learning methods which are CNN and ensemble learning have been used and 95\% average accuracy has been obtained for each model, for binary classification and $78 \%$ and $75 \%$ average accuracy has been obtained for each model respectively for multi class classification problem.
\end{abstract}

Keywords: Bacterial Pneumonia, CNN, Deep Learning, Ensemble Learning, Pneumonia, Smote Method, Viral Pneumonia.

\section{Introduction}

More than 1 million people get pneumonia every year in the U.S Unfortunately, 50,000 of these people die from this disease. Many factors such as age, smoking, alcohol use, and malnutrition can cause people to have pneumonia. Pneumonia can be seen in people of different age groups, but it is more risky due to low immune systems in two-year-old and younger babies and individuals aged 65 and over [1]. Pneumonia can be a disease that can be managed with early diagnosis and treatment.

Chest X-ray images are used for the diagnosis of pneumonia. But the diagnosis of pneumonia from chest $\mathrm{X}$-ray images is a difficult task even for specialist radiologists. The appearance of pneumonia in X-ray images is often uncertain, it can be confused with other diseases and behave like many other benign abnormalities. These inconsistencies have caused important subjective decisions and variations among radiologists in the diagnosis of pneumonia [2].

\footnotetext{
${ }^{I}$ Istanbul Technical University, Department of Electronics and Communication Engineering., Istanbul, TURKEY; ORCID ID : 0000-0002-0943-9381

${ }^{2}$ Istanbul Technical University, Department of Electronics and Communication Engineering, Istanbul, TURKEY; ORCID ID : 0000-0001-7660-3236

${ }^{3}$ Istanbul Technical University, Department of Electronics and Communication Engineering, Istanbul, TURKEY; ORCID ID : 0000-0001-6124-2394

* Corresponding Author Email: darici18@itu.edu.tr
}

Therefore, computerized support systems are needed to help radiologists diagnose pneumonia from chest X-ray images.

In medical imaging, the correct diagnosis or evaluation of a disease depends on both image acquisition and image interpretation. Image acquisition has recently begun to take advantage of computer technology, as image acquisition has improved significantly in recent years and devices are collecting data at faster and higher resolution [3]. Thanks to large data sets, rapid progress is made in computerized vision problems based on deep learning. Object recognition, perception and segmentation performance results were found to give better results compared to manual diagnosis. In deep learning, radiology imaging has generally shown similar performance improvements in the field of medical image analysis for the detection and segmentation tasks of human anatomical or pathological structures [4].

Machine learning and deep learning which are the sub-branches of artificial intelligence used in many fields in recent years also offer hope to help experts in early diagnosis of diseases. Studies show that deep learning gives good results in the field of health.

In [5] Siar et al. tried to detect the brain tumor using Magnetic Resonance Imaging (MRI) images with the CNN method. With the fully connected layer of softmax used in the last stage, they have reached an accuracy rate of $98.67 \%$ in detecting the disease. Based on the high performance achieved in $\mathrm{CNN}$, they provided feature extraction of the images with machine learning methods and then used these images in CNN. This new used method yielded 99.12\% in the test set. 
Yoo et al. developed a CNN-based automated pipeline to diagnose prostate cancer. In this study, data set from 427 patients were used and the area under the ROC curve was determined as 0.87 [6].

In [7] Kieu et al. developed multiple CNN structures to detect normal and abnormal thorax images. In this method, they divided the entrance of $\mathrm{CNN}$ into three parts, namely right, left and whole of a thorax image. They gave all these parts to $\mathrm{CNN}$ and finally combined the results of these $\mathrm{CNN}$ models with what they call the Fusion rule. They demonstrated the applicability of the multiple CNN method they proposed with a $96 \%$ result.

Wang et al. [8] have developed a deep CNN method used to detect 14 thorax diseases, which they call Thorax-Net. This method consists of classification and attention section. The classification branch uses a classifier-feature extractor network to save users from the hassle of manual feature extraction and classification. Using the attention branch class tags and pathological abnormalities, it analyzes the feature extraction map obtained from the classification branch. Thorax-Net tries to achieve a result by centering the outputs of these two branches. Wang et al. With the Thorax-net architecture, they have seen that the area below the average ROC per class has reached 0.7876 .

There are also many studies based on deep learning related to the diagnosis of pneumonia. For example; Ayan et al. [4] emphasized the importance of early diagnosis of pneumonia disease in their study. They used the VGG-16 and Xception models in the detection of the disease through transfer learning and model adaptation methods. 624 chest X-ray images used in the testing phase, they reached $87 \%$ accuracy for the VGG-16 model and $82 \%$ accuracy for the Xception model. They also added that the Xception model gives better results than the Inception-V3 model. In [9], Talo used the Res-Net152 model with the learning transfer method for the detection of pneumonia disease. It achieved $97.4 \%$ recognition success from the data set used without any pretreatment and feature extraction.

O'Quinn et al. tried to detect the presence of the disease using 5659 images in DICOM format. It used $70 \%$ of the data set for train and the rest for testing. Alexnet achieved $76 \%$ recognition success in the method used by the deep learning network with transfer learning [10].

In another study [11], Varshni et al. made a comparison for the diagnosis of pneumonia between different models based on CNN, which they used through transfer learning as feature extraction, and different classifiers they used as classifiers. With their statistical results, they show that pre-trained $\mathrm{CNN}$ models used in conjunction with supervised classifier algorithms can be very useful in analyzing chest x-ray images, especially to detect Pneumonia. According to these results, they stated that they obtained the best results when using DenseNet-169 for feature extraction and Support Vector Machines (SVM) as classifier.

Stephen et al. unlike studies based on transfer learning used in pneumonia diagnosis, they trained the chest data set in CNN created from scratch. They increased the data set with data enhancement methods such as flip horizontal, change width and height, zoom, etc. The CNN model used has 4 convolution layers with $3 \times 3$ different number of filters, and ReLu activation function and maximum pooling layer after each convolution layer. The last two layers are fully connected layers. Using the sigmoid function after the fully connected layer, it was determined whether the image was pneumonia or normal. The accuracy of the model has been tested with different dimensions of the image and the highest performance for the 200x200 color image has been reached as $93.73 \%[12]$.
Urey et al. processed the data set in three different ways. These preprocesses are increase in color space, increase in contrast and opening the image. They have tried different combinations of these preprocesses for different networks. In their last attempt, they used $\mathrm{CNN}$ model to create a feature extraction map of the pre-processed $\mathrm{X}$-ray images using contrast enhancement and image unpacking during the feature extraction phase. And this model has been tested with two different classification techniques. While the first method is artificial neural network, the second model that gives the highest performance is ResNet architecture. The model that gives the highest performance in their studies correctly determines the pneumonia disease by $78.73 \%$ [13].

In [14] Hammoudi et al. have worked on deep learning models that can be used to diagnose pneumonia in order to contribute to healthcare workers using radiographic images. They used different types of deep learning methods such as ResNet34, ResNet50 and DenseNet169, VGG-19 and a dual use model (Inception ResNetV2 - RNN). They have tried to classify chest X-ray images into viral pneumonia, bacterial pneumonia and normal.

\section{Deep Learning}

There has been much talk about all the possibilities of machines that have recently learned to do what people do in factories, warehouses, offices and homes. Along with the developing technology, terms such as artificial intelligence, machine learning and deep learning are also on the agenda.

In short, Artificial Intelligence is the science of making computers smart. The purpose of Artificial Intelligence is to make computers more useful by understanding the nature of intelligence [15]. Deep learning methods are used for fast learning and implementation of complex data by disabling human factor from machine learning [16]. Deep learning is a machine learning branch based on a set of algorithms that attempt to model high level abstractions in data using multiple processing layers with complex structures or in other ways [17].

\subsection{Convolutional Neural Network}

The term deep learning refers to multi layered artificial neural networks (ANN). Recently, deep learning has become one of the most powerful tools in the literature as it can process large amounts of data [18]. The Convolutional Neural Networks (CNN) is one of the most known deep learning networks. Significant achievements have been achieved in studies such as voice recognition, image processing, pattern recognition using CNN. CNN is a deep neural network in which at least one of the layers forming it, feature extraction is performed by applying the convolution process [19]. $\mathrm{CNN}$ model has different layers as given below

1)Input Layer: The Input Layer forms the first layer of CNN. In the input layer, the input is given raw to the network. In order for the computer to understand the given input image, the pixels of the input are converted into numerical data.

2)Convolution Layer: The convolution layer is also known as the feature extraction layer because the property of the given image in the network is extracted in this layer. The feature map of the image is drawn using various filters in the convolution layer [20]. The filters used can be of different sizes, such as $2 \times 2,3 \times 3,5 \times 5$. In this layer, the convolution process is applied to the input data using given filters. There are some hyperparameters such as zero padding and stride used in convolution process. Convolution starts from the upper left corner of the input matrix and then continues by sliding the filter to the right and down. Stride specifies the number of steps in this scroll. When stride is 1 , the filters are moved one by one. 
When it is 2 , the filters take two steps while shifting. If the number of steps is high, it means that the output will be smaller as a result of the convolution. Generally the number of steps 1 or 2 is preferred in CNN. The Figure 1. below shows how the output size changes when the stride is 1 or 2 .

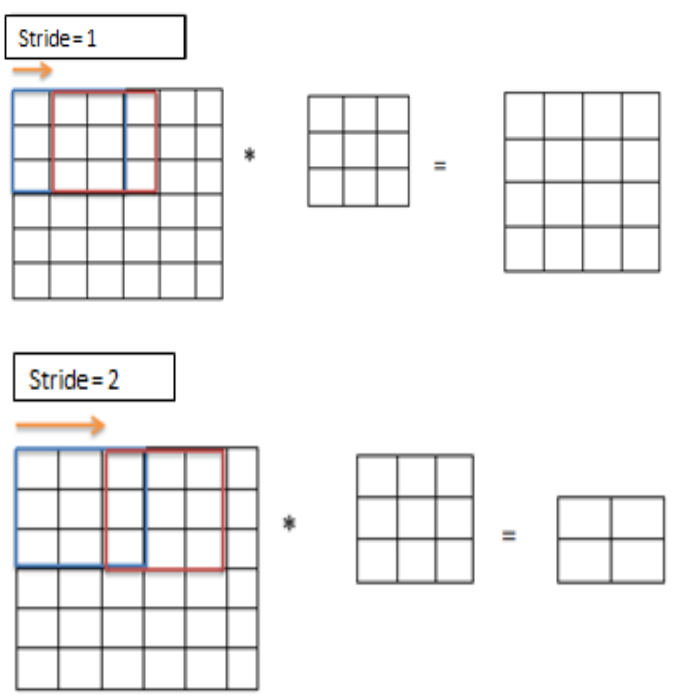

Fig. 1 Effect of stride to the output in the convolution process.

Sometimes it may be necessary to fill the border of the input matrix with zeros. The purpose of using the zero padding is to control the size of the output matrix in convolution layer. As shown in the Fig 2., a $5 \times 5$ size matrix is obtained as a result of the convolution process applied by a $3 \times 3$ size filter, and a $2 \times 2$ size matrix when zero padding is not applied.

3)Pooling Layer: The pooling layer is generally used to reduce the number of parameters and complexity in a CNN model. For the next convolution layer, it decreases the input size without affecting the depth size. So, the height and width values of the input decrease after pooling, while its depth does not change. There are some different types of pooling layers but the most popular one is max pooling layer. A filter size and number of stride are determined at maximum pooling. Depending on this parameters, the maximum value inside the filter becomes the new value of the output.

4)Fully Connected Layer: The fully connected layer comes after some convolution, activation function of ReLU and pooling layers in CNN. For the fully connected layer, the feature map is converted to the 1D feature vector which is called "Flattening". This layer is called the fully connected layer because it is connected to all neurons of the previous layer. This layer helps the produce output from extracted features from previous layers and then pass it to the output layer where each neuron has a classification probability of labels.

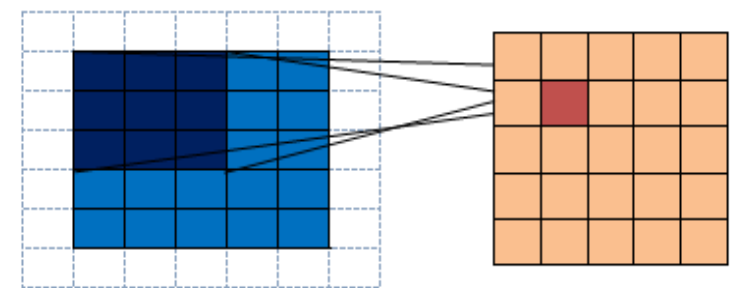

(a)

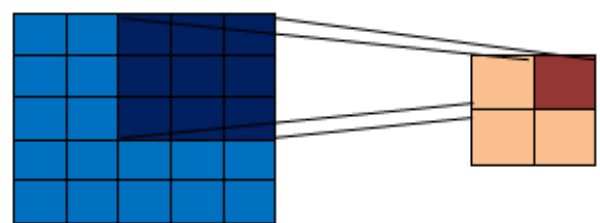

(b)

Fig. 2 The output of convolution process when (a) zero padding is applied ,(b) zero padding is not applied.

\subsection{Ensemble Learning}

Ensemble learning is an effective and increasingly adopted technique that combines different learning algorithms to improve the overall performance of the model [21]. Unlike ordinary machine learning approaches that try to learn a hypothesis from training data, ensemble learning methods try to create a series of hypotheses and combine them to use [22]. Ensemble learning consists of three different methods:

1) Bagging: It stands for 'bootstrap aggregating'. It was proposed by Breiman [23] to combine predictive results of models trained on randomly distributed training sets to improve classification performance [24]. As seen in Fig 3, training sets are randomly distributed in bagging method. Training and prediction are carried out for each training set. And these prediction results are combined at the end.

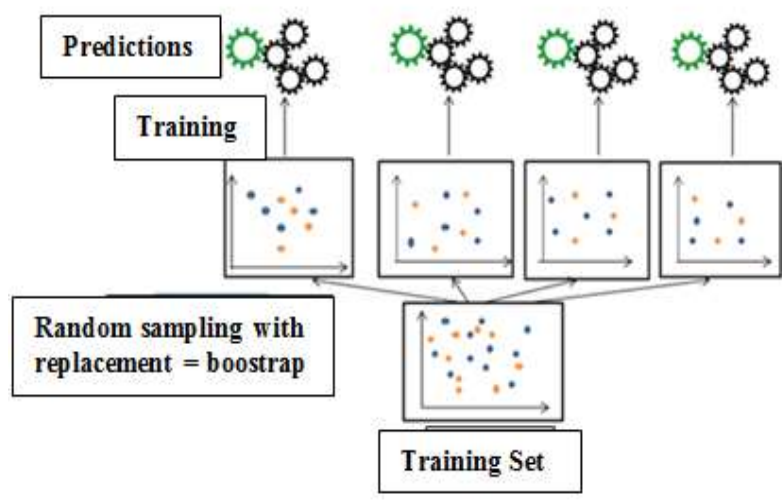

Fig. 3 Bagging method .

2) Boosting : Boosting is the algorithm that combines a number of weak classifiers to create a strong classifier [24]. This method creates a gradually recurring community. As in Fig 4., each new predictive model tries to increase performance by emphasizing the incorrect classifications of the previous model [24]. Adaboosting [25] is still the most used boosting method, although many different boosting methods have been developed.

3)Majority Voting : The majority voting method is a method that improves the performance of the classifier by voting [21]. In this method, different classifiers are used as shown in Fig 5. and each classifier makes a prediction. The estimates of the classifiers are looked at and the prediction with the most votes is considered as the prediction of the model. Since three of the four different classifiers used here estimate the sample used as $\mathrm{X}$, the sample is considered an estimated $\mathrm{X}$ as a result 


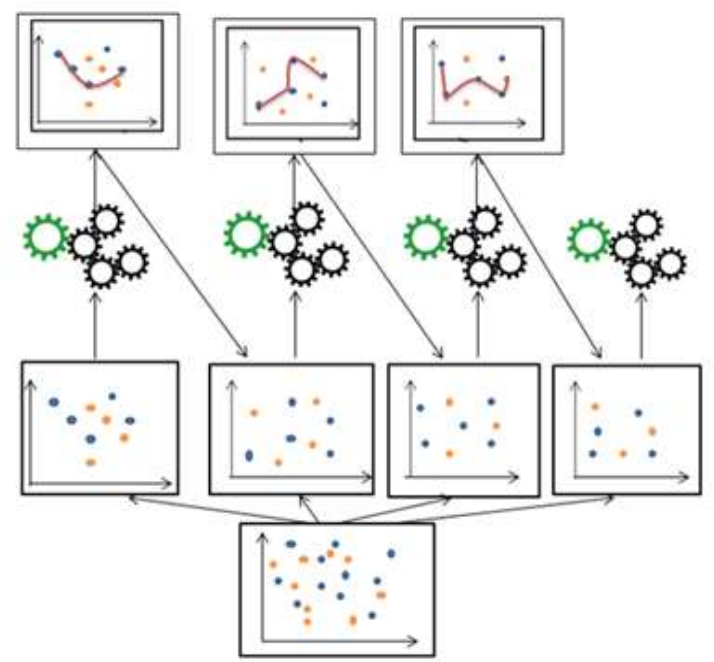

Fig. 4 Boostig method .

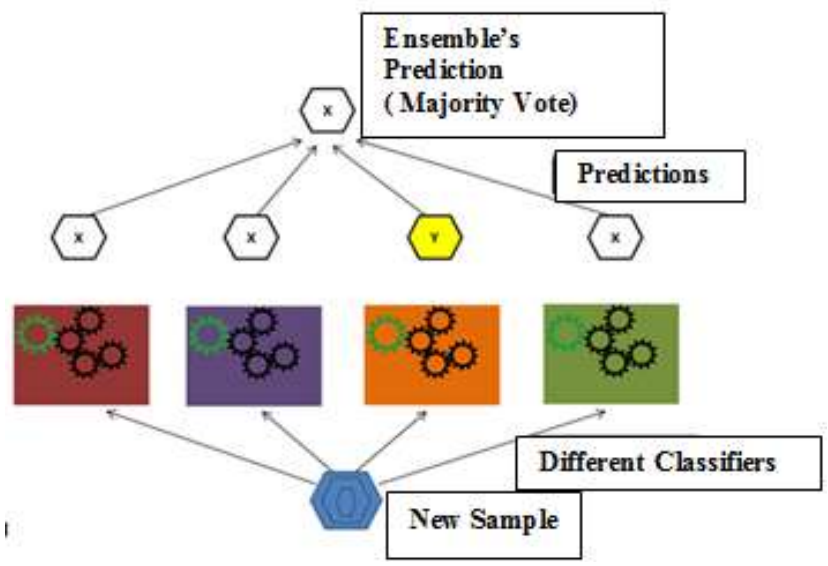

Fig. 5 Majority Voting method .

\section{Materials and Methods}

\subsection{Outline of Methodology}

This study consists of two parts: binary classification (Pneumonia/Normal) and multi-class classification (Bacterial Pneumonia/Viral Pneumonia/Normal) of Chest X-ray images. For each classification type, $\mathrm{CNN}$ and Ensemble Learning methods were used separately.

\subsection{Dataset}

The used dataset ${ }^{1}$ in which images of Chest X-ray have been labeled as normal and pneumonia, consists of Chest X-ray images from one to five year old children provided by the Guangzhou Women's and Children's Health Center. This dataset has been obtained Of these images low-quality or illegible ones have been eliminated and the remaining images have been graded by a total of three experts for use in artificial intelligence studies. For multi class classification problem, pneumonia class is divided into two classes which are bacterial pneumonia and virus/viral pneumonia. There are 5840 images in total, each of which is different in size. Normally the dataset has an unbalance distribution over classes.
The number of images in each class were balanced with the SMOTE method in order to prevent the inclination towards the class which has majority, during the training. SMOTE method is an over-sampling approach in which the minority class is oversampled by creating "synthetic" examples to make the image distribution in classes balanced. To create synthetic samples using SMOTE method, firstly a sample should be chosen from dataset. Then the difference between chosen sample and its nearest neighbor is calculated. This difference is multiplied by a random number between $0-1$. At the end, this value is added to the first chosen sample. These steps are repeated for the number of nearest neighbors. In [26] SMOTE method has been applied for different types of data for different purposes. After SMOTE method which is applied on only training data to balance number of data in each class, dataset has 6808 for training and 1168 for testing for binary classification . For multi class classification, after SMOTE method training set has 6714 and test set has $1168 \mathrm{X}$-ray images. The samples of Chest X-ray dataset for each class can be seen in Fig.6.

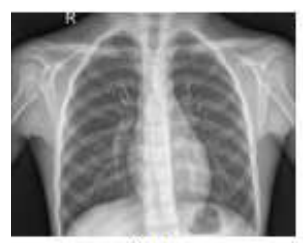

(a)

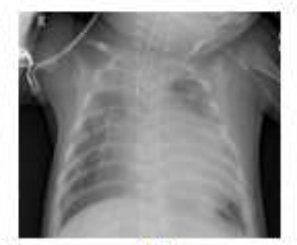

(b)

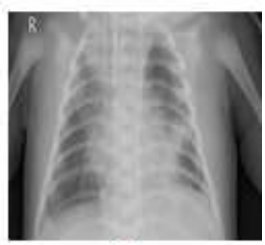

(c)
Fig 6. Chest X-Ray samples; (a) Normal, (b) Virus ,(c) Bacteria

\subsection{Proposed Model Convolutional Neural Network (CNN)}

In $\mathrm{CNN}$ model used for this work, separable convolution process has been applied for each convolution layer except first layer instead of ordinary convolution. The feature that distinguishes the separable convolution from the classical convolution is that it is separable. The separable convolution is divided into depth convolution and point convolution. This method makes the model has less parameters and decreases the computational time. CNN model starts with two convolutional process with $163 \times 3$ filter. ReLU activation follows each convolution layer. Also zero padding has been applied for convolution processes. After convolution layers, max pooling has been used. For the other layers, separable convolutions have been applied with ReLU and zero padding using different number of $3 \times 3$ filters. Similarly, max pooling follows the convolution layers. However, differently batch normalization has been used before max pooling. Batch normalization is proposed as a technique that helps coordinate the updating of multiple layers in the model. Same processes have been seen in the following block. For last two layers before fully connected layer, dropout process has been applied after max pooling. The drop out process removes neurons below a certain threshold at each training stage to prevent the network overfitting. All the weights of last layer are connected to fully connected layer. In this work, there are three fully connected layers, each followed by dropout. Finally, all the weights pass through a Softmax function which gives the probability of the output for classes. For each classification problems which are binary and multi class, same CNN model has been used, also stride is one for each 
convolution layers and batch size is 16 , number of epoch is 20 and input RGB image is resized to $112 \times 112$ before training.

\subsection{Proposed Model Ensemble Learning}

For this proposed model using majority voting, three different CNN models have been used within Ensemble Learning for each classification problem. First model is CNN model mentioned in Part C. Second model is another $\mathrm{CNN}$ model which has convolution layers using filters with $3 \times 3$ size and ReLU, batch normalization after each convolution layer and dropout layer. Outputs of last layer are connected to output layer in which Softmax function is used. The last model is Inception-V3. Inception-V3 is a deep learning model. For the first layers of this model, weights of the pre-trained model with ILSVRC ImageNet [27] dataset which has 1000 classes have been used. This process called as transfer learning. In the last layer, a fully connected layer was used for this study. The output of this layer is given to the Softmax function. After the training processes of these three models, the final predictions obtained using the majority voting method were the results of this ensemble learning model. Same as the CNN model, batch size and number of epoch of each model used in Ensemble Learning is 16 and 20 respectively. Proposed CNN model and Ensemble Learning model in this work shown in Fig. 7.
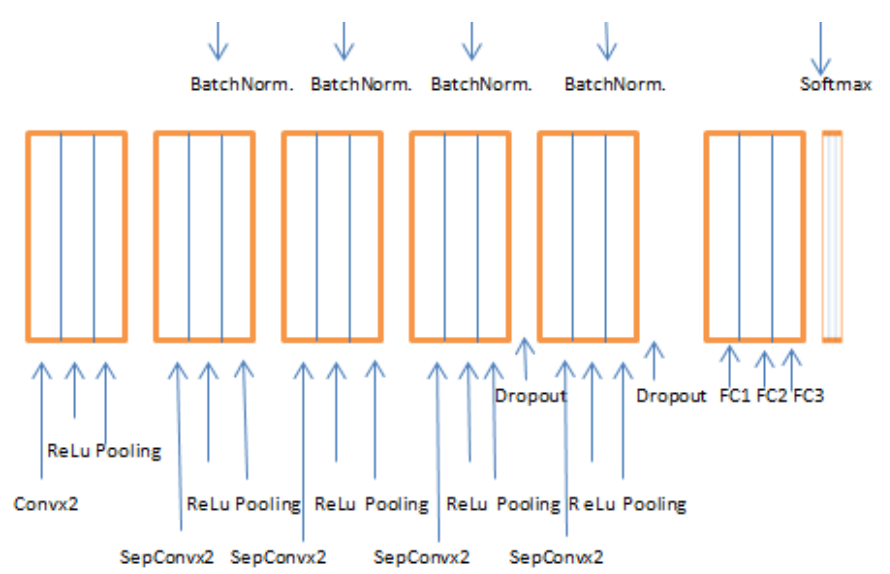

(a)

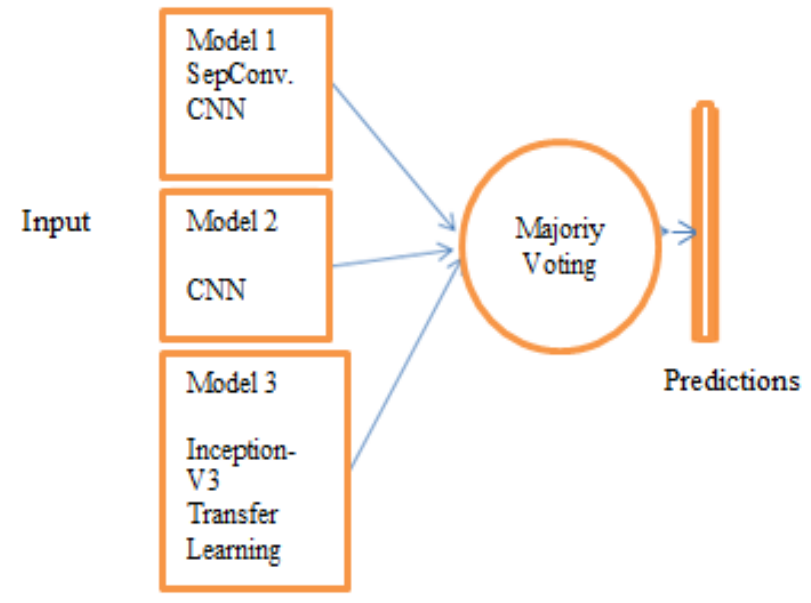

(b)

Fig.7 Proposed models (a) CNN model, (b) Ensemble Learning model.

\section{Experimental Results}

\subsection{Classification Performance}

The performance of this work for each model is evaluated in the Accuracy, Precision, Recall and F1 score.

$$
\begin{aligned}
& \text { Accuracy }=\frac{T P+T N}{T P+T N+F N+F P} \\
& \text { Precision }=\frac{T P}{T P+F P} \\
& \text { Recall }=\frac{T P}{T P+F N} \\
& F 1 \text { score }=2 \frac{\text { Precision } \times \text { Recall }}{\text { Precision }+ \text { Recall }}
\end{aligned}
$$

Where TP is the number of images correctly classified. FP is the number of images misclassified to some other classes, TN is the number of images correctly classified which does not belong to that class. FN denotes number of images which belongs to some classes but misclassified to another class. In both models used for this study, 95\% accuracy rate has been achieved for binary classification. For the multi class classification, the average precision, recall and $\mathrm{f} 1$ scores for the CNN model are $80 \%, 78 \%$, $78 \%$ and $77 \%, 75 \%, 75 \%$ respectively for the Ensemble model. Considering the tables below, both models proposed for this study give higher performance in classification than most of the other studies. In multi class classification, when we look at the average accuracy values for each class one by one, some results give higher performance than the transfer learning methods in the other proposed study. It can be seen experimental results clearly

for binary and multi class classification for each model in Table 1, $2,3,4$.

Table 1. Results of CNN model for binary classification

\begin{tabular}{|l|c|c|c|}
\hline Class \Metrics & Precision & Recall & F1 score \\
\hline Pneumonia & 0.98 & 0.97 & 0.97 \\
\hline Normal & 0.92 & 0.94 & 0.93 \\
\hline Average & 0.95 & 0.95 & 0.95 \\
\hline
\end{tabular}

Table 2. Results of Ensemble Learning model for binary classification

\begin{tabular}{|l|l|l|l|}
\hline Class $\backslash$ Metrics & Precision & Recall & F1 score \\
\hline Pneumonia & 0.97 & 0.97 & 0.97 \\
\hline Normal & 0.91 & 0.93 & 0.92 \\
\hline Average & 0.94 & 0.95 & 0.95 \\
\hline
\end{tabular}

Table 3. Results of CNN model for multiclass classification

\begin{tabular}{|l|c|c|c|}
\hline Class\Metrics & Precision & Recall & F1 score \\
\hline Normal & 0.91 & 0.94 & 0.93 \\
\hline Virus P. & 0.74 & 0.48 & 0.58 \\
\hline Bacterial P. & 0.75 & 0.90 & 0.82 \\
\hline Average & 0.80 & 0.78 & 0.78 \\
\hline
\end{tabular}

Table 4. Results of Ensemble Learning model for multiclass classification

\begin{tabular}{|l|c|c|c|}
\hline Class \Metrics & Precision & Recall & F1 score \\
\hline Normal & 0.84 & 0.93 & 0.88 \\
\hline Virus P. & 0.74 & 0.43 & 0.54 \\
\hline Bacterial P. & 0.75 & 0.89 & 0.81 \\
\hline Average & 0.77 & 0.75 & 0.75 \\
\hline
\end{tabular}

\subsection{Comparision}

There are a lot of works about pneumonia detection as a binary classification. However most of them are based on transfer learning approaches. Instead of using trained weights, we created a deep learning model from strach for both classification problem. 
Table 5 and 6 show comparision results of models for binary and multi class classification.

Table 5. Comparision of model results for binary classification.

\begin{tabular}{|l|l|l|}
\hline Method & Techniques & Accuracy \\
\hline Ayan et al.[2] & Vgg-16 & 0.87 \\
\hline Talo [9] & ResNet152 & 0.97 \\
\hline O'Quinn et al [10] & Alexnet & 0.76 \\
\hline Stephen et al[12] & CNN & 0.93 \\
\hline Urey et al. [13] & Resnet & 0.78 \\
\hline $\begin{array}{l}\text { This work model1 / } \\
\text { model 2 }\end{array}$ & CNN / Ensemble & $0.95 / 0.95$ \\
\hline
\end{tabular}

Table 6. Comparision of model results for multiclass classification.

\begin{tabular}{|l|l|c|c|c|}
\hline & & \multicolumn{3}{c|}{ Accuracy \% } \\
\hline \multicolumn{1}{|c|}{ Method } & Techniques & Bacteria & Virus & Normal \\
\hline $\begin{array}{l}\text { Hammoudi et } \\
\text { al[14] }\end{array}$ & Vgg19 & 87.84 & 81.08 & 79.05 \\
\hline $\begin{array}{l}\text { Hammoudi et } \\
\text { al[14] }\end{array}$ & $\begin{array}{l}\text { Resnet\&RNN } \\
1\end{array}$ & 86.49 & 84.46 & 63.52 \\
\hline $\begin{array}{l}\text { Hammoudi et } \\
\text { al[14] }\end{array}$ & $\begin{array}{l}\text { Resnet\&RNN } \\
2\end{array}$ & 90.54 & 84.46 & 63.52 \\
\hline $\begin{array}{l}\text { Hammoudi et } \\
\text { al[14] }\end{array}$ & Densenet169 & 97.97 & 96.62 & 92.57 \\
\hline This work & CNN & 90.07 & 48 & 94.46 \\
\hline This work & Ensemble & 88.95 & 43 & 93.48 \\
\hline
\end{tabular}

\section{Conclusion}

As a conclusion in this study two different deep learning models which are CNN and Ensemble learning have been proposed for binary and multi class classification problems. Same dataset has been used for both problems. SMOTE method which is one of the over sampling methods used to deal with imbalance data problems has been used to make the image distribution in classes equal in the dataset. The classes are Pneumonia and Normal for the binary classification, on the other hand, these are Virus Pneumonia, Bacterial Pneumonia and Normal for multi class classification. Some metrics which are precision, recall and F1 score and accuracy have been used to show the results. As a result, both proposed models reached $95 \%$ accuracy rate for binary classification and average precision, recall and F1 score are 80\%, $78 \%, 78 \%$ and $77 \%, 75 \%, 75 \%$ for multi class classification for $\mathrm{CNN}$ and Ensemble model respectively. This shows that CNN model is better than ensemble model for multi class classification. In addition, these models are trained on Google Colab which provides free GPU, in less than half an hour. Also, our proposed model which is CNN has only $6 \mathrm{M}$ parameters, on the other hand, proposed model in [14] which is DenseNet169 has 14M parameters which means there is a huge difference. Number of parameters is one the affects which effects computational time. Results show that SMOTE method can be used for also Chest X-ray images and different types of deep learning models can be used to reach high accuracy rates instead of using pre-trained networks. Since chest $\mathrm{X}$ ray images are difficult to distinguish even for radiologists, these results may be higher if more labelled X-ray datasets are obtained in the future.

\section{References}

[1] Health Topics Pneumonia [Online] Available: https://www.nhlbi.nih.gov/health-topics/pneumonia

[2] E. Ayan and H. M. Ünver, "Diagnosis of Pneumonia from Chest XRay Images Using Deep Learning," 2019 Scientific Meeting on Electrical-Electronics \& Biomedical Engineering and Computer Science (EBBT), Istanbul, Turkey, 2019, pp. 1-5, doi:

\subsection{9/EBBT.2019.8741582.}

[3] H. Greenspan, B. van Ginneken and R. M. Summers, "Guest Editorial Deep Learning in Medical Imaging: Overview and Future Promise of an Exciting New Technique," in IEEE Transactions on Medical Imaging, vol. 35, no. 5, pp. 1153-1159, May 2016, doi: 10.1109/TMI.2016.2553401

[4] X. Wang, Y. Peng, L. Lu, Z. Lu, M. Bagheri and R. M. Summers, "ChestX-Ray8: Hospital-Scale Chest X-Ray Database and Benchmarks on Weakly-Supervised Classification and Localization of Common Thorax Diseases," 2017 IEEE Conference on Computer Vision and Pattern Recognition (CVPR), Honolulu, HI, 2017, pp. 3462-3471, doi: 10.1109/CVPR.2017.369.

[5] M. Siar and M. Teshnehlab, "Brain Tumor Detection Using Deep Neural Network and Machine Learning Algorithm," 2019 9th International Conference on Computer and Knowledge Engineering (ICCKE), Mashhad, Iran, 2019, pp. 363-368.

[6] S.Yoo, I. Gujrathi , M.A. Haider ,F. Khalvati "Prostate Cancer Detection using Deep Convolutional Neural Networks" ,A Nature Research Journal, Scientific Reports volume 9, Article number: 19518 (2019)

[7] P. N. Kieu, H. S. Tran, T. H. Le, T. Le and T. T. Nguyen, "Applying Multi-CNNs model for detecting abnormal problem on chest x-ray images," 2018 10th International Conference on Knowledge and Systems Engineering (KSE), Ho Chi Minh City, 2018, pp. 300-305.

[8] H. Wang, H. Jia, L. Lu and Y. Xia, "Thorax-Net: An Attention Regularized Deep Neural Network for Classification of Thoracic Diseases on Chest Radiography," in IEEE Journal of Biomedical and Health Informatics, vol. 24, no. 2, pp. 475-485, Feb. 2020.

[9] M. Talo, "Pneumonia Detection from Radiography Images using Convolutional Neural Networks," 2019 27th Signal Processing and Communications Applications Conference (SIU), Sivas, Turkey, 2019, pp. 14.

[10] W. O'Quinn, R. J. Haddad and D. L. Moore, "Pneumonia Radiograph Diagnosis Utilizing Deep Learning Network," 2019 IEEE 2nd International Conference on Electronic Information and Communication Technology (ICEICT), Harbin, China, 2019, pp. 763-767.

[11] D. Varshni, K. Thakral, L. Agarwal, R. Nijhawan and A. Mittal, "Pneumonia Detection Using CNN based Feature Extraction," 2019 IEEE International Conference on Electrical, Computer and Communication Technologies (ICECCT), Coimbatore, India, 2019, pp. 1-7.

[12] O. Stephen , M. Sain ,U. J. Maduh , D. Jeong, “An Efficient Deep Learning Approach to Pneumonia Classification in Healthcare", Hindawi Journal of Healthcare Engineering,Volume 2019,Article ID 4180949, 7 pages

[13] D.Y.Urey, C. J. Saul, C.D.Taktakoglu , "Early Diagnosis of Pneumonia with Deep Learning",2019, http://arxiv.org/abs/ 1904.00937

[14] K. Hammoudi1,H. Benhabiles, M.Melkemi, F. Dornaika, I Arganda-Carreras, D.e Collard,A.Scherpereel, "Deep Learning on Chest X-ray Images to Detect and Evaluate Pneumonia Cases at the Era of COVID-19”, arXiv:2004.03399, Apr 2020

[15] Kuşçu, E. (2015). Çeviride Yapay Zeka Uygulamaları . Atatürk Üniversitesi Kazım Karabekir Eğitim Fakültesi Dergisi , 0 (30), 45 58 . Retrieved from https://dergipark.org.tr/tr/pub/ataunikkefd/ issue $/ 2789 / 37502$

[16] Kayaalp, K., \& Süzen, A. A. (2018). Derin Öğrenme ve Türkiye'deki Uygulamaları. IKSAD Publishing House.pp.5-6.

[17] D. K. Wornyo,F. B.Kataka1,(2016). A Review of Deep Machine Learning. International Journal of Engineering Research in Africa. 24. $124-136.10 .4028$

[18] Albawi, Saad \& Abed Mohammed, Tareq \& ALZAWI, Saad (2017). Understanding of a Convolutional Neural Network.

[19] Zeki Kuş, "Mikrokanonikal Optimizasyon Algoritmasi Ile Konvolüsyonel Sinir Ağlarinda Hiper Parametrelerin Optimize Edilmesi”, Fatih Sultan Mehmet Vakif Üniversitesi Lisansüstü 
Eğitim Enstitüsü/Yüksek Lisans Tezi, Haziran 2019.

[20] Y. Guo, Y. Liu, A. Oerlemans, S. Wu, and M. S. Lew, (2015). Deep learning for visual understanding: A review. Neurocomputing.

[21] A. Rojarath, W. Songpan and C. Pong-inwong, "Improved ensemble learning for classification techniques based on majority voting," 2016 7th IEEE International Conference on Software Engineering and Service Science (ICSESS), Beijing, 2016, pp. 107-110, doi: 10.1109/ICSESS.2016.7883026.

[22] Z.-H. Zhou, "Ensemble learning," in Encyclopedia Biometrics, S. Z. Li and A. Jain, Eds. New York, NY, USA: Springer, 2015, pp. 411-416.

[23] W. Liu, M. Zhang, Z. Luo and Y. Cai, "An Ensemble Deep Learning Method for Vehicle Type Classification on Visual Traffic Surveillance Sensors," in IEEE Access, vol. 5, pp. 24417-24425, 2017, doi: 10.1109/ACCESS.2017.2766203.

[24] Ferreira A.J., Figueiredo M.A.T. (2012) Boosting Algorithms: A Review of Methods, Theory, and Applications. In: Zhang C., Ma Y. (eds) Ensemble Machine Learning. Springer, Boston, MA. https://doi.org/10.1007/978-1-4419-9326-7_2

[25] L. Wang, M. Sugiyama, C. Yang, Z.-H. Zhou, and J. Feng, “'On the margin explanation of boosting algorithms," in Proc. COLT, 2008, pp. 479-490

[26] N. V. Chawla, K. W. Bowyer, L. O. Hall, W. P. Kegelmeyer, "SMOTE: Synthetic Minority Over-sampling", Technique Journa of Artificial Intelligence Research 16 (2002) 321-357 Submitted 09/01; published 06/02

[27] Deng, J., Dong, W., Socher, R., Li, L.J., Li, K. and Fei-Fei, L. (2009). ImageNet: A large-scale hierarchical image database, Computer Vision and Pattern Recognition, 2009. CVPR 2009. IEEE Conference on, pp.248-255 